University of Nebraska - Lincoln

DigitalCommons@University of Nebraska - Lincoln

$5-7-2004$

\title{
Effect of interface bonding on spin-dependent tunneling from the oxidized Co surface
}

\author{
Kirill D. Belashchenko \\ University of Nebraska-Lincoln, belashchenko@unl.edu \\ Evgeny Y. Tsymbal \\ University of Nebraska-Lincoln, tsymbal@unl.edu \\ Mark van Schilfgaarde \\ Arizona State University, mark.van_schilfgaarde@kcl.ac.uk \\ Derek A. Stewart \\ Sandia National Laboratories, Livermore, California, stewart@cnf.cornell.edu \\ Ivan I. Oleynik \\ University of South Florida, oleynik@cas.usf.edu \\ See next page for additional authors
}

Follow this and additional works at: https://digitalcommons.unl.edu/mrsecfacpubs

Part of the Materials Science and Engineering Commons

Belashchenko, Kirill D.; Tsymbal, Evgeny Y.; van Schilfgaarde, Mark; Stewart, Derek A.; Oleynik, Ivan I.; and Jaswal, Sitaram, "Effect of interface bonding on spin-dependent tunneling from the oxidized Co surface" (2004). Faculty Publications: Materials Research Science and Engineering Center. 15.

https://digitalcommons.unl.edu/mrsecfacpubs/15

This Article is brought to you for free and open access by the Materials Research Science and Engineering Center at DigitalCommons@University of Nebraska - Lincoln. It has been accepted for inclusion in Faculty Publications: Materials Research Science and Engineering Center by an authorized administrator of DigitalCommons@University of Nebraska - Lincoln. 


\section{Authors}

Kirill D. Belashchenko, Evgeny Y. Tsymbal, Mark van Schilfgaarde, Derek A. Stewart, Ivan I. Oleynik, and Sitaram Jaswal 


\title{
Effect of interface bonding on spin-dependent tunneling from the oxidized Co surface
}

\author{
K. D. Belashchenko, ${ }^{1}$ E. Y. Tsymbal, ${ }^{1}$ M. van Schilfgaarde, ${ }^{2}$ D. A. Stewart, ${ }^{3}$ I. I. Oleynik, ${ }^{4}$ and S. S. Jaswal ${ }^{1}$ \\ ${ }^{1}$ Department of Physics and Astronomy and Center for Materials Research and Analysis, University of Nebraska, \\ Lincoln, Nebraska 68588, USA \\ ${ }^{2}$ Department of Chemical and Materials Engineering, Arizona State University, Tempe, Arizona 85287, USA \\ ${ }^{3}$ Sandia National Laboratories, Livermore, California 94551, USA \\ ${ }^{4}$ Department of Physics, University of South Florida, Tampa, Florida 33620, USA
}

(Received 4 December 2003; published 7 May 2004)

\begin{abstract}
We demonstrate that the factorization of the tunneling transmission into the product of two surface transmission functions and a vacuum decay factor allows one to generalize Jullière's formula and explain the meaning of the "tunneling density of states" in some limiting cases. Using this factorization we calculate spin-dependent tunneling from clean and oxidized fcc Co surfaces through vacuum into Al using the principallayer Green's-function approach. We demonstrate that a monolayer of oxygen on the Co(111) surface creates a spin-filter effect due to the Co-O bonding which produces an additional tunneling barrier in the minority-spin channel. This changes the minority-spin dominated conductance for the clean Co surface into a majority-spin dominated conductance for the oxidized Co surface.
\end{abstract}

DOI: 10.1103/PhysRevB.69.174408

PACS number(s): 72.25.Mk, 73.23.-b, 73.40.Gk, 73.40.Rw

\section{INTRODUCTION}

Spin-dependent tunneling (SDT) in magnetic tunnel junctions (MTJ's) is a dynamically developing area of research that attracted a lot of attention due to promising applications in nonvolatile random access memories and nextgeneration magnetic field sensors (for a recent review of SDT see Ref. 1). The experimental efforts have succeeded in achieving large reproducible tunneling magnetoresistance in MTJ's ${ }^{2}$ but also raised fundamental questions regarding the nature of SDT. One such question is the role of the ferromagnet/insulator interfaces in controlling the spin polarization (SP) of the tunneling conductance defined as $P$ $=\left(G_{\uparrow}-G_{\downarrow}\right) /\left(G_{\uparrow}+G_{\downarrow}\right)$, where $G_{\sigma}=\left(e^{2} / h\right) \Sigma_{\mathbf{k}_{\|}} T_{\sigma}\left(\mathbf{k}_{\|}\right)$is the conductance for spin channel $\sigma, T_{\sigma}$ is the transmission function, and $\mathbf{k}_{\|}$is the transverse component of the wave vector.

Commonly the expected spin dependence of the tunneling current is deduced by considering the symmetry of the Bloch states in the bulk ferromagnetic electrodes and the complex band structure of the insulator. ${ }^{3,4}$ By identifying those bands in the electrodes that are allowed by symmetry to couple efficiently to the evanescent states decaying most slowly in the barrier one can predict the SP of the conductance. However, this approach has two deficiencies. First, it assumes that the barrier is sufficiently thick so that only a small focused region of the surface Brillouin zone (SBZ) contributes to the tunneling current. For realistic MTJ's with a barrier thickness of about $1 \mathrm{~nm}$ this assumption is usually unjustified. Second, symmetry considerations alone applied to bulk materials are not always sufficient to predict the SP. It is critical to take into account the electronic structure of the ferromagnet/ barrier interfaces which, as it was shown both experimentally ${ }^{5}$ and theoretically, ${ }^{6}$ controls SDT.

An important mechanism by which the interfaces affect the SP of the tunneling current is the bonding between the ferromagnetic electrodes and the insulator. ${ }^{7}$ This effect was put forward to explain positive and negative values of tun- neling magnetoresistance depending on the applied voltage in MTJ's with $\mathrm{Ta}_{2} \mathrm{O}_{5}$ and $\mathrm{Ta}_{2} \mathrm{O}_{5} / \mathrm{Al}_{2} \mathrm{O}_{3}$ barriers ${ }^{8}$ and to elucidate the inversion of the $\mathrm{SP}$ in $\mathrm{Co} / \mathrm{SrTiO}_{3} /$ $\mathrm{La}_{0.67} \mathrm{Sr}_{0.33} \mathrm{MnO}_{3}$ MTJ's. ${ }^{9}$ So far there are no theoretical studies explaining the microscopic origin of this phenomenon.

In this paper we report the results of first-principles calculations of SDT from clean and oxidized Co surfaces through vacuum into $\mathrm{Al}$ and demonstrate the crucial role of the bonding between $\mathrm{Co}$ and $\mathrm{O}$ atoms at the surface. This system was chosen for investigation because the MTJ's based on alumina have predominantly O-terminated $\mathrm{Co} / \mathrm{Al}_{2} \mathrm{O}_{3}$ interfaces. ${ }^{10}$ By replacing alumina by vacuum we can ignore the complexity of the atomic structure of the amorphous alumina and focus on effects of surface oxidation. Moreover, this system can be directly studied using spin-polarized STM. ${ }^{11}$

We show that a monolayer of oxygen on the Co surface creates a spin-filter effect due to the Co-O bonding by producing an additional tunneling barrier in the minority-spin channel. This reverses the sign of the SP from negative for the clean Co surface to positive for the oxidized Co surface revealing the decisive role of interface bonding in SDT.

\section{SURFACE TRANSMISSION FUNCTIONS}

We approach the tunneling problem in the spirit of perturbation theory. ${ }^{12}$ We consider the system consisting of "left" and "right" leads separated by a relatively thick barrier and assume that two-dimensional translational periodicity in transverse directions is preserved for both electrodes including their surfaces (although it may be different for each electrode). Each Bloch wave with a transverse wave vector $\mathbf{k}_{\|}^{L}$ coming from the left lead has a decay tail in the vacuum composed of the waves with transverse wave vectors $\mathbf{k}_{\|}^{L}$ $+\mathbf{G}_{i}$ where $\mathbf{G}_{i}$ are the reciprocal-lattice vectors of the SBZ of the left lead. ${ }^{13}$ At sufficient distances from the surface (typically just a few lattice parameters for low-index sur- 

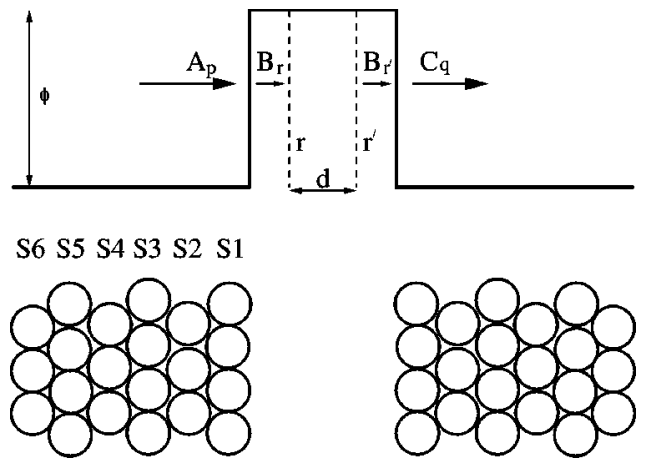

FIG. 1. Geometry of a tunnel junction with a vacuum barrier. The top graph schematically shows the potential barrier for electrons at the Fermi level. Dashed lines show the positions of the reference planes assigned to the electrodes for the calculation of the surface transmission functions. Each scattering state is described by four amplitudes: $A_{p}$ of the incoming Bloch wave; $B_{r}, B_{r}^{\prime}$ of the surviving evanescent wave at the reference planes assigned to the left and right electrodes; and $C_{q}$ of the transmitted Bloch wave in the right electrode (there may be several transmitted waves). The bottom scheme depicts atomic layers in the electrodes and their labeling used in the text.

faces) all waves with $\mathbf{G}_{i} \neq 0$ vanish and may be neglected. On the other hand, the wave vector $\mathbf{k}_{\|}^{R}$ is conserved at the right lead surface. This means that $\mathbf{k}_{\|}$is conserved across the entire system even if there is no common in-plane periodicity and $\mathbf{k}_{\|}^{L}, \mathbf{k}_{\|}^{R}$ are defined with respect to different SBZ's. In this case, each tunneling eigenstate is almost identical to an evanescent plane wave in the central region of the barrier.

For a given $\mathbf{k}_{\|}$the transmission function is the sum of the transmission coefficients for all tunneling eigenstates corresponding to all incoming Bloch waves with this $\mathbf{k}_{\|}$in the left electrode. At the same time, each transmission coefficient for a given eigenstate contains a sum over outgoing states in the right electrode with the same $\mathbf{k}_{\|}$. Let us choose a reference plane in the vacuum region at a sufficient distance from the surface of an electrode, so that the eigenstates for all $\mathbf{k}_{\|}$are already indistinguishable from the barrier eigenstates at this plane (see Fig. 1). For each tunneling eigenstate the amplitude of the barrier eigenstate between the reference planes is the only parameter coupling the left and right electrodes. Then, the $S$-matrix element coupling the states in the two electrodes may be written as

$$
S_{p q}^{\sigma}=S_{p r}^{\sigma} S_{r r^{\prime}} S_{r^{\prime} q}^{\sigma},
$$

where $p$ is the incoming and $q$ the outgoing Bloch state in the left and right lead, respectively, $r$ and $r^{\prime}$ denote the same vacuum eigenstate at the left and right reference planes, and we omitted the dependence on $\mathbf{k}_{\|}$for all the $S$-matrices for brevity. The vacuum $S$-matrix $S_{r r}$, simply describes the exponential decay of the wave function in the vacuum. Note that no summation is implied in Eq. (1), because the state $r$ is uniquely defined by $\mathbf{k}_{\|}$. The simple product of $S$-matrices in Eq. (1) without any multiple-scattering terms is a consequence of our assumption that the barrier is sufficiently thick. Thus, we see that the transmission function $T\left(\mathbf{k}_{\|}\right)$of the MTJ is factorized:

$$
T_{\sigma}\left(\mathbf{k}_{\|}\right)=t_{L}^{\sigma}\left(\mathbf{k}_{\|}\right) \exp \left[-2 \kappa\left(\mathbf{k}_{\|}\right) d\right] t_{R}^{\sigma}\left(\mathbf{k}_{\|}\right) .
$$

Here we replaced $\left|S_{r r^{\prime}}\right|^{2}$ by its explicit exponential form with

$$
\kappa\left(\mathbf{k}_{\|}\right)=\left(\frac{2 m \phi}{\hbar^{2}}+k_{\|}^{2}\right)^{1 / 2}
$$

where $\phi$ is the work function, $d$ is the distance between reference planes assigned to the electrodes as shown in Fig. 1. All information about the properties of individual surfaces is described by the surface transmission functions (STF's) $t_{L}^{\sigma}, t_{R}^{\sigma}$ :

$$
t_{L}\left(\mathbf{k}_{\|}\right)=\sum_{p}\left|\frac{B_{r}}{A_{p}}\right|^{2}, \quad t_{R}\left(\mathbf{k}_{\|}\right)=\sum_{q}\left|\frac{C_{q}}{B_{r^{\prime}}}\right|^{2},
$$

where the four amplitudes characterize the behavior of tunneling eigenstates at the two surfaces (see Fig. 1). The different definitions of $t_{L}$ and $t_{R}$ are due to the fact that they pertain to different boundary conditions. Specifically, $t_{L}$ and $t_{R}$ stem from the matching of Bloch wave functions with the vacuum eigenstates, decaying and growing into the vacuum, respectively. The definition of $t_{L}$ implies the solution of a scattering problem for the incoming wave with amplitude $A_{p}$, and $B_{r}$ is the amplitude of this scattering eigenstate at the reference plane in the vacuum. On the other hand, $t_{R}$ describes an inverse scattering problem in which the exponentially decaying wave in the vacuum with amplitude $B_{r^{\prime}}$ at the reference plane is scattered on the right surface; here $C_{q}$ is the amplitude of the outgoing Bloch state $q$ in the right electrode for this "eigenstate." Note that physically, this state is forbidden because it grows to infinity in the vacuum, but it is still a formal solution of the Schrödinger equation with the specified boundary condition at infinity.

Each surface may be considered as forming the left or the right interface of the junction. It is straightforward to show that, just as in the case of a transmission matrix connecting the propagating states in the two electrodes,${ }^{14}$ the requirement of flux conservation results in the reciprocity condition $t_{L}\left(\mathbf{k}_{\|}\right)=t_{R}\left(\mathbf{k}_{\|}\right) \equiv t\left(\mathbf{k}_{\|}\right)$for any (laterally periodic) surface, as long as an appropriate normalization of the wave functions is chosen. Specifically, all Bloch waves in the electrodes should be normalized for unit flux, while the vacuum eigenstates should be normalized for unit "imaginary flux" $\kappa / m$. The easiest way to establish this reciprocity condition is to prove it for free electrons in a semi-infinite potential well, and then to use this free-electron system as a second electrode in a junction. Since the total transmission function for a twoelectrode system is reciprocal, ${ }^{14}$ this proves the above reciprocity condition for the STF. Note that this proof does not rely on time-reversal symmetry, because time reversal also replaces $\mathbf{k}_{\|}$by $-\mathbf{k}_{\|}$. However, this symmetry gives another useful relation $t\left(\mathbf{k}_{\|}, \mathbf{H}\right)=t\left(-\mathbf{k}_{\|},-\mathbf{H}\right)$ where $\mathbf{H}$ is the external magnetic field.

It is easy to see from Eq. (4) with the chosen unit flux normalization that the STF is proportional to the Fermi-level value of the $\mathbf{k}_{\|}$- and energy-resolved charge density for the 
given spin, which is generated by the incoming Bloch states and taken at the reference plane (any localized surface states are excluded). Indeed, the energy-resolved charge density may be written as

$$
\rho_{\sigma}(\mathbf{r}, E)=\frac{1}{N_{\|}} \sum_{\mathbf{k}_{\|}} \rho_{\sigma}\left(\mathbf{r}, \mathbf{k}_{\|}, E\right)
$$

where the $\mathbf{k}_{\|}$- and energy-resolved charge density is

$$
\rho_{\sigma}\left(\mathbf{r}, \mathbf{k}_{\|}, E\right)=\sum_{p}\left|\psi_{\sigma \mathbf{k}_{\|} p}^{F}(\mathbf{r})\right|^{2}
$$

where the summation is over the incoming Bloch states with the given $\mathbf{k}_{\|}$and $E$, and the superscript $F$ emphasizes that the scattering eigenstates $\psi_{\mathbf{k}_{\mid} p}^{F}$ are normalized so that the incoming Bloch waves carry unit flux normal to the interface. Setting $A_{p}=1$ in Eq. (4), we see that $t\left(\mathbf{k}_{\|}\right)$is given by Eq. (6) where $\mathbf{r}$ is taken at the reference plane [since we neglect all components with $\mathbf{G}_{i} \neq 0$ at the reference plane, $\rho\left(\mathbf{r}, \mathbf{k}_{\|}, E\right)$ does not depend on $\mathbf{r}_{\|}$, the location within this plane].

Although we considered a vacuum tunneling barrier, the analysis can be extended to other physically important cases of insulating barriers. Indeed, the main requirement for the validity of Eqs. (1) and (2) is that the complex band structure be predominantly carried by a single evanescent wave for each $\mathbf{k}_{\|}$. At a minimum, this premise must hold for the "active" regions of the SBZ that contribute appreciably to the tunneling current. This means that other tunneling states must have a notably larger imaginary part of the wave vector compared to the dominant one. In the case of a vacuum barrier, this amounts to the neglect of all vacuum eigenstates with $\mathbf{G}_{i} \neq 0$. In practice this criterion is well satisfied for sufficiently thick wide-gap tunnel barriers, such as $s p$-bonded oxides. However, for any particular barrier, this assumption has to be carefully verified by checking the complex band structure of the barrier for the presence of additional slowly decaying states in the active regions of the SBZ. It is important to note that the area of the active region quickly shrinks as the barrier thickness is increased, ${ }^{4}$ indicating that the single evanescent state criterion will often lead to an additional requirement of "sufficient thickness" of the barrier. Note that this requirement is not related to a similar one stemming from the neglect of backscattering.

The factorization (2) allows us to study tunneling between completely different electrodes. For practical purposes, the STF can be calculated using $T_{\sigma}\left(\mathbf{k}_{\|}\right)$obtained for a symmetric MTJ by factoring out the vacuum decay factor for the given choice of reference planes, and then taking the square root. The resulting STF's for different surfaces may then be convolved with an appropriate vacuum decay factor, exp $(-2 \kappa d)$, to obtain the transmission functions for asymmetric MTJ's. In particular, $T_{\sigma}\left(\mathbf{k}_{\|}\right)$for the antiparallel magnetization of the two electrodes (and the tunneling magnetoresistance) can be found from the up- and down-spin STF's calculated from the transmission function for the parallel magnetization.

\section{GENERALIZATION OF JULLIÈRE'S FORMULA}

Tunneling magnetoresistance $R$ is often discussed in terms of Jullière's formula ${ }^{15}$

$$
R=\frac{2 P_{L} P_{R}}{1-P_{L} P_{R}}
$$

where $P_{L}, P_{R}$ are the "spin polarizations" of the left and right electrodes. Equation (7) may be derived assuming that $G^{\sigma} \propto \rho_{L}^{\sigma} \rho_{R}^{\sigma}$, where $\rho_{L}^{\sigma}, \rho_{R}^{\sigma}$ are the "tunneling densities of states" of the electrodes, and the spin polarization is defined as $P=\left(\rho^{\uparrow}-\rho^{\downarrow}\right) /\left(\rho^{\uparrow}+\rho^{\downarrow}\right)$. The popularity of this formula is due to the fact that it usually agrees reasonably well with experiment, as long as the spin polarizations, which are directly related to the tunneling densities of states, are taken from Meservey-Tedrow experiments ${ }^{16}$ with the same barrier as that used in the given MTJ. However, the validity of Jullière's formula has been debated for a long time, and the reasons for its apparent agreement with experiment are unclear. The physical meaning of the tunneling density of states is also unclear, but it is obvious both from elementary quantum mechanics ${ }^{17}$ and from experiments that the tunneling properties of a magnetic heterostructure are determined not by the ferromagnet alone, but rather by the ferromagnet/ barrier combination and by the structure of the interface. A number of explicit first-principles calculations for idealized MTJ's without disorder confirmed this fact (see, e.g., Ref. 18). However, it was suggested ${ }^{19}$ that phase decoherence due to disorder which is always present in realistic MTJ's may recover the factorization of the tunneling conductance in a product of transport densities of states, which are essentially equal to the regular densities of states at the surfaces of the electrodes if there are no resonant localized states in the barrier. Moreover, it was shown ${ }^{20}$ within a single-band tight binding-model that in the limit of strong disorder one recovers Jullière's formula (7) by identifying $P_{L}, P_{R}$ with the measurable spin polarizations of the tunneling current for the same electrode/barrier systems. Therefore, it seems that there are good reasons for the widespread use of Jullière's formula, and it is highly desirable to elucidate these reasons.

Let us explore the connection between Eq. (2) and Jullière's formula (7). In Eq. (2) the simple product of the tunneling densities of states is replaced by a convolution of STF's, which explicitly include the effects of bulk densities of states and of the surface structure. Thus we can consider Eq. (2) as a generalization of Jullière's formula for an ideal MTJ with no disorder.

However, we may go further and identify limiting cases where Eq. (2) can be directly related to Jullière's formula, providing formal definitions of the tunneling densities of states of the electrodes appropriate for these cases. First, consider the case of a disordered insulating barrier. Such a barrier may be characterized by its eigenstates, half of which are decaying from left to right, and the other half from right to left. Although these eigenstates do not have a conserved $\mathbf{k}_{\|}$ anymore, it is still clear that tunneling will be dominated by Feynman paths that do not "loop back," because each path carries a weight decaying exponentially with its length (see below). Therefore, we may still write an expression similar 
to Eq. (1) neglecting backscattering, but now we should sum up over all barrier eigenstates (now defined in real space). Within this formulation $S_{r r^{\prime}}$ is still diagonal because it describes the decay of a single eigenstate.

The weight of a Feynman path in the imaginary-time functional integral often used for tunneling problems ${ }^{21}$ (with Euclidean action written in its reduced Maupertuis form; see, e.g., Ref. 22) is given by $\exp \left[-\int \kappa(l) d l\right]$ up to a prefactor, where the integral is taken along the path, $\kappa=[2 m(V$ $-E)]^{1 / 2}$ and $V(\mathbf{r})$ is the potential. In an ordered insulator many paths with similar weights contribute to the path integral resulting in the formation of the complex band structure. However, in a disordered insulator the tunneling current may be dominated by Feynman paths running close to a relatively small number of "easy" paths with locally maximum weights, i.e., by imaginary-time classical paths. ${ }^{21,22}$ If there is only one such channel or one class of channels with similar properties (e.g., due to surface roughness), Eq. (2) will produce Jullière's formula where $\rho^{\sigma}$ is simply the Fermilevel value of the energy-resolved charge density given by Eq. (6) integrated over $\mathbf{k}_{\|}$and taken at some reference point within the channel. (Now each term describes the scattering eigenstate corresponding to the single incoming Bloch wave with the given $\mathbf{k}_{\|}$.) Like STF, this quantity does not depend on the properties of the other side of the barrier. This conclusion agrees with the results of Ref. 20 showing that the tunneling current through a strongly disordered barrier is dominated by a small number of random configurations, and that Jullière's formula is also recovered in this limit.

Now consider the case when disorder is weak close to the interfaces, but remains strong in the insulator. Obviously, the $S$-matrix of the disordered insulator in $\mathbf{k}_{\|}$representation will be essentially a random matrix, and after averaging Eq. (2) thus yields Jullière's formula with $\rho^{\sigma} \propto \Sigma_{\mathbf{k}_{\|}} t^{\sigma}\left(\mathbf{k}_{\|}\right)$. This case is the easiest from the computational point of view, because the STF's may be directly calculated for a $\mathbf{k}_{\|}$-conserving MTJ.

It is instructive to compare this result with the conclusions of Mathon and Umerski ${ }^{19}$ on the applicability of Jullière's formula obtained using the transfer Hamiltonian formalism. Our approach shares in common with Ref. 19 the neglect of multiple reflections across the junction. However, the assumption of constant matrix elements (hopping integrals) for all Bloch waves made in Ref. 19 completely removes all physical effects connected with orbital- and spin-dependent bonding at interfaces. This obviously contradicts the experimental findings showing that the spin polarization of the tunneling current and magnetoresistance strongly depend on the type of barrier used. ${ }^{1}$ In our approach, the STF's for the electrodes allow us to encapsulate the effects of the interface structure and provide the proper dependence of the tunneling current on barrier type. Jullière's formula obtained in the limiting case of full decoherence inside the insulator is expressed in terms of the spin polarization actually measured in the Meservey-Tedrow experiment (assuming that the superconductor acts as an ideal, nonbiased spin detector).

Finally, for very thick $\mathbf{k}_{\|}$-conserving barriers the tunneling current may be carried predominantly by a close vicinity of some special $\mathbf{k}_{\|}$points in the SBZ (e.g., the $\Gamma$ point). In this case, the tunneling density of states is simply equal to the value of $T\left(\mathbf{k}_{\|}\right)$at this $\mathbf{k}_{\|}$.

It is not clear a priori whether any one of these three limiting cases is directly applicable to realistic MTJ's, although it seems that disorder in the insulator together with the "channelization" of the tunneling current are both likely to play a major role. However, the emergence of Jullière's formula in these different scenarios suggests that it may actually have a rather wide range of applicability. In general, the tunneling density of states should be identified with some appropriately averaged energy-resolved charge density taken at the Fermi level at a sufficient distance from the interface within the barrier. Unlike the bulk density of states, this function fully takes into account the relevant properties of the surface.

\section{TUNNELING FROM CLEAN AND OXIDIZED Co(111) SURFACES THROUGH VACUUM INTO Al}

We calculated the transmission functions using the principal-layer Green's-function approach ${ }^{23}$ based on the tight-binding linear muffin-tin orbital method (TB-LMTO) in the atomic sphere approximation (ASA) and the transmission matrix formulation of Ref. 24. Local density approximation (LDA) was used in all calculations. All atomic potentials were determined self-consistently using the supercell approach within the TB-LMTO-ASA method. The vacuum barrier was modeled using empty spheres in the positions corresponding to the continuation of the crystal lattice of the electrodes. We have also performed full-potential LMTO calculations $^{25}$ which confirmed all main features of the band structure of the oxidized $\operatorname{Co}(111)$ surface discussed below.

We checked the validity of factorization (2) by calculating $T_{\sigma}\left(\mathbf{k}_{\|}\right)$for (100)- and (111)-oriented fcc Co electrodes with parallel magnetizations, taking the square root, and convolving $t_{\uparrow}\left(\mathbf{k}_{\|}\right)$with $t_{\downarrow}\left(\mathbf{k}_{\|}\right)$. Then, the result was compared with the independent calculation for the antiparallel configuration in a range of energies. The agreement was always excellent (better than 1\%), except for a couple of specific energies for a (100) MTJ with four vacuum "monolayers" (ML) where narrow resonances appear in the minority channel. ${ }^{26}$ If the vacuum barrier is extended to $8 \mathrm{ML}$, excellent agreement is restored.

Using the factorization (2) we investigated the SP of the conductance from ferromagnetic electrodes to a nonmagnetic material, $\mathrm{Al}(111)$, which served as a detector of the tunneling SP in the spirit of the Meservey-Tedrow experiments. ${ }^{16}$ As expected, the calculated STF of Al is free-electron-like, having almost perfect Gaussian shape originating from the vacuum decay factor up to the reference plane. Therefore, this surface may be considered as equally transparent for all Bloch waves, and the total transmission function for a MTJ with Al spin-detector electrode is essentially a product of the other electrode's STF and the vacuum decay factor.

First, we discuss the properties of a Co/vacuum/Al MTJ with a clean $\operatorname{Co}(111)$ surface. Figures 2(a) and 2(b) show the $\mathbf{k}_{\|}$-resolved transmission for the majority- and minority-spin electrons within the SBZ of $\operatorname{Co}(111)$. The Fermi surface of 


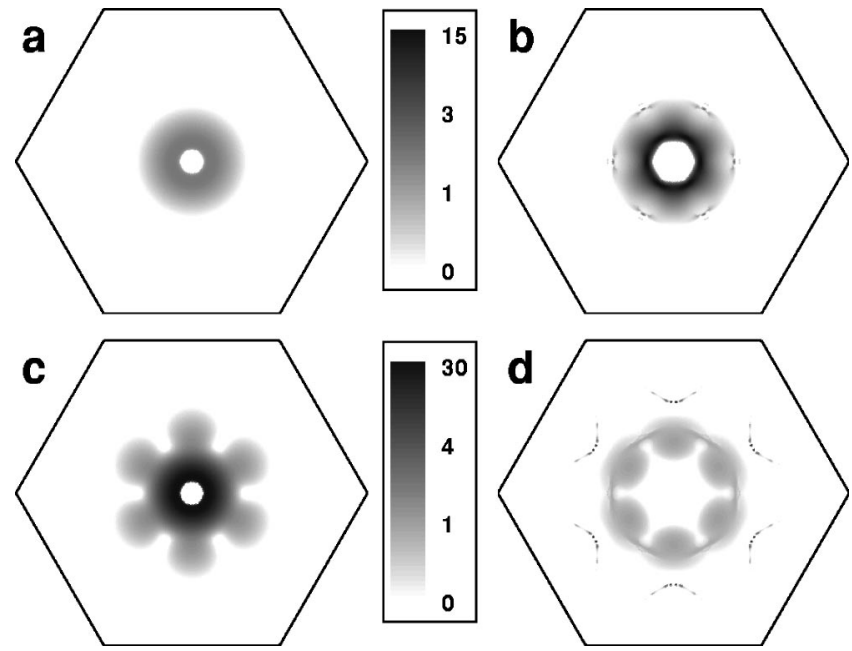

FIG. 2. $\mathbf{k}_{\|}$-resolved transmission (logarithmic scale) from clean and oxidized $\mathrm{Co}(111)$ surfaces through vacuum into Al. (a) Clean surface, majority spin. (b) Clean surface, minority spin. (c) Oxidized surface, majority spin. (d) Oxidized surface, minority spin. The vacuum layer thickness is $2 \mathrm{~nm}$ for clean and $1.7 \mathrm{~nm}$ for oxidized Co surface. The first surface Brillouin zone is shown. Units are $10^{-11}$ for (a), (b) and $10^{-14}$ for (c), (d).

Co viewed along the [111] direction has holes close to the $\bar{\Gamma}$ point with no bulk states in both spin channels, which results in zero conductance in this area. The majority-spin transmission [Fig. 2(a)] varies rather smoothly and is appreciable over a relatively large area of the SBZ. On the other hand, the minority-spin transmission [Fig. 2(b)] has a narrow crown-shaped "hot ring" around the edge of the Fermisurface hole. The analysis of layer and $\mathbf{k}_{\|}$-resolved density of states (DOS) shows that it is not associated with surface states, ${ }^{26}$ but is related to the enhancement of bulk $\mathbf{k}_{\|}$-resolved DOS near the Fermi-surface edge [compare Fig. 2(b) with Fig. 4(c)].

As seen from Figs. 2(a) and 2(b) the Fermi-surface hole is smaller for majority spins. Therefore, the conductance should become fully majority-spin polarized in the limit of very thick barriers. However, since the Fermi-surface hole is also quite narrow for minority spins, positive SP is only achieved at very large barrier thicknesses $d \sim 10 \mathrm{~nm}$, while for typical values of $d \sim 2 \mathrm{~nm}$ the SP is about $-60 \%$ and depends weakly on $d$.

The oxidized Co surface was modeled by an $\mathrm{O}$ monolayer placed on top of the $\operatorname{Co}(111)$ electrode. The equilibrium atomic structure of this surface was found using the pseudopotential plane-wave method ${ }^{27}$ within the generalized gradient approximation. We used both types of stacking: $A B C A$ and $A B C B$, where the last symbol designates the position of the $\mathrm{O}$ monolayer. The $\mathrm{O}$ atoms were assumed to lie in symmetric positions above the second (S2) or first (S1) subsurface Co layer, respectively (these layers are shown in Fig. 1 in the absence of the $\mathrm{O}$ monolayer). We allowed the $\mathrm{O}$ layer and two Co layers (S1 and S2) to relax in the direction normal to the surface, while the positions of atoms in deeper layers $(\mathrm{S} 3, \ldots)$ were kept fixed. The energies of both equilibrium configurations of oxygen monolayers were found to
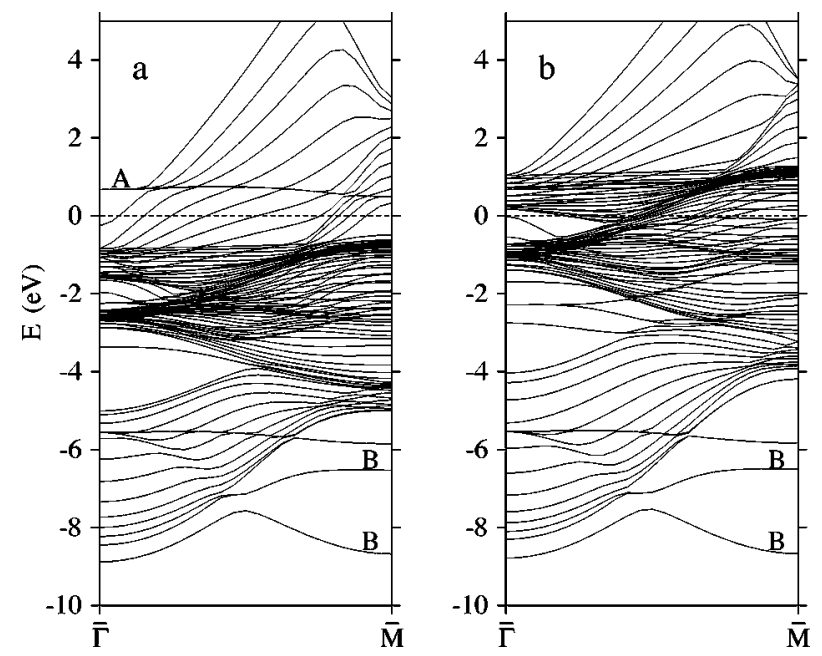

FIG. 3. Band dispersions along the $\bar{\Gamma} \bar{M}$ direction for (a) majority- and (b) minority-spin electrons. Energy is referenced from $E_{F}$. The bonding Co-O surface bands are marked $B$, and the pure antibonding surface band is marked $A$.

be very close to each other. All results of interest in the present context are quite similar for these two stackings. Below all specific data are given for the $A B C B$ stacking. The equilibrium interlayer distances were found to be $2.14 \AA$ between layers S3 and S2, $2.18 \AA$ between S2 and S1, and $1.08 \AA$ between $\mathrm{S} 1$ and O layers, compared to $2.07 \AA$ between the adjacent Co layers in the bulk. The $\mathrm{Co}-\mathrm{O}$ bond length is thus $1.82 \AA$.

Presence of oxygen at the surface of cobalt raises the question of whether electron correlations similar to those characteristic for transition-metal oxides may be strong enough to induce significant changes in the band structure at the surface. However, the enhancement of correlations in oxides is due to much weaker screening of Coulomb interaction compared to the metallic state. On the other hand, cobalt atoms below the oxygen monolayer preserve the closepacked configuration of bulk cobalt except that the three nearest neighbors out of 12 are absent. Therefore, it is reasonable to expect that screening of Coulomb interaction in the $3 d$ shell is not much weaker compared to the bulk. For this reason, we believe that LDA electronic structure of the oxidized Co surface is correct as far as the main features are concerned.

The oxygen monolayer dramatically changes the electronic structure of the underlying Co layer making this layer almost magnetically dead. This change can be understood from band dispersion plots shown in Fig. 3. For each spin, the free-standing oxygen monolayer would have three energy bands deriving from $2 p$ states, each doubly degenerate due to $\sigma_{z}$ reflection symmetry ( $z$ is the axis normal to the surface). When the monolayer is deposited onto the Co surface, the degeneracy is lifted, and two sets of three bands each are formed corresponding to bonding and antibonding mixing of oxygen and cobalt orbitals. The three bonding bands (marked $\mathrm{B}$ in Fig. 3) lie well below the bulk Co $3 d$ band, whereas the antibonding states are close to the Fermi energy $E_{F}$. As a result of this bonding the local DOS for the S1 layer at $E_{F}$ is 

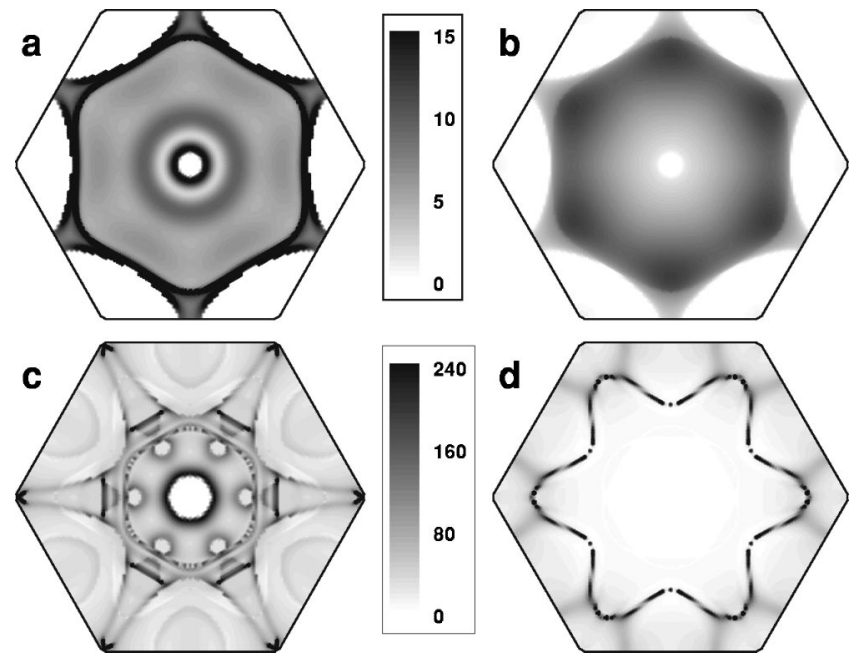

FIG. 4. $\mathbf{k}_{\|}$-resolved local DOS at $E_{F}$ (arbitrary units) for the oxidized Co surface: (a) S6 layer, majority spin; (b) S1 layer, majority spin; (c) S6 layer, minority spin; (d) S1 layer, minority spin.

strongly reduced, so that, according to the Stoner criterion, magnetism in this layer is almost completely suppressed. The magnetic moment of $\mathrm{Co}$ atoms in the $\mathrm{S} 1$ layer is only $0.17 \mu_{B}$.

Transmission of propagating bulk states from the electrode through the barrier is very sensitive to the degree of mixing of these states with the antibonding surface states. This mixing is controlled by a selection rule which follows from the fact that all bands can be classified as "even" or "odd" according to their symmetry with respect to $\bar{\Gamma} \bar{M}$ reflection. Although this classification is exact only along the $\bar{\Gamma} \bar{M}$ direction, it is approximately valid throughout the entire SBZ.

According to this classification, two of the three surface bands are even, and one is odd. On the other hand, the freeelectron-like band of bulk Co which forms the only Fermisurface sheet for majority-spin electrons is even, while the minority-spin states on the Fermi-surface sheet closest to the $\bar{\Gamma}$ point are odd. Even and odd bands are orthogonal and cannot mix.

This selection rule results in the principal difference between the majority- and minority-spin transmission. The majority-spin bands are shown in Fig. 3(a). One even and one odd antibonding surface bands (marked A) are degenerate at the $\bar{\Gamma}$ point (at about $0.8 \mathrm{eV}$ above $E_{F}$ ). At a short distance from the $\bar{\Gamma}$ point both bands enter the continuum of bulk states. The odd band does not mix with the bulk states along the $\bar{\Gamma} \bar{M}$ line and remains almost flat due to repulsion from a lower-lying band. On the other hand, the even band readily mixes with the free-electron-like majority-spin band crossing the Fermi level and completely loses its surface localization. This is evident from Figs. 4(a) and 4(b) which show the $\mathbf{k}_{\|}$-resolved DOS of majority-spin electrons for the bulklike S6 and surface S1 Co layers. The DOS for the S1 layer [Fig. 4(b)] is appreciable in the entire area of the SBZ where bulk states are available and has no sharp features that might indicate localized surface states. This implies that the bulk majority-spin states extend to the very surface of the electrode and therefore can readily tunnel through the barrier.

The situation is very different for minority-spin states. Although the odd surface band is again almost flat and lies above $E_{F}$, the even surface band crossing the Fermi level does not mix with the odd minority-spin band. As a consequence, the $\mathbf{k}_{\|}$-resolved DOS for $\mathrm{Co}(\mathrm{S} 1)$ layer [Fig. 4(d)] is large only along the curve lying at the periphery of the SBZ where the Co-O antibonding surface band crosses the Fermi level (oxygen DOS looks very similar). As a result, the bulk minority-spin states responsible for most tunneling transmission from the clean surface only extend up to the S2 layer, encountering a band gap in the S1 and oxygen layers. Thus, an additional tunneling barrier is introduced in the minorityspin channel, and the total SP of the tunneling transmission becomes almost $100 \%$ positive, which is evident from Figs. 2(c) and 2(d).

The predicted effect of interface bonding is not limited only to the $\operatorname{Co}(111)$ surface. We have also calculated the transmission from clean and oxidized $\mathrm{Co}(100)$ and $\mathrm{Ni}(111)$ electrodes and found that surface oxidation reverses the SP due to the bonding between $\mathrm{Co}$ or $\mathrm{Ni}$ and $\mathrm{O}$. As it was shown earlier, the reversal of the SP also occurs for the $\mathrm{Fe}(100)$ surface. ${ }^{28}$

\section{CONCLUSION}

We have shown that the problem of calculating the transmission function for a sufficiently thick insulating barrier is reduced to the solution of three separate problems, namely the penetration of the bulk wave functions into the barrier from both sides, and the behavior of the evanescent barrier eigenstates. This separation provides a natural generalization of Jullière's formula. We identified three limiting cases when the original Jullière's formula is recovered. The tunneling density of states in this formula is identified with an appropriately averaged energy-resolved charge density generated by the bulk Bloch states within the barrier and taken at the Fermi level.

Using the factorization of the transmission function into a product of surface transmission functions and a barrier decay factor we calculated the spin polarization of the tunneling current from clean and oxidized $\mathrm{Co}(111)$ surfaces through vacuum into $\mathrm{Al}$. We showed that the bonding between Co and $\mathrm{O}$ atoms at the oxidized surface controls the spindependent tunneling by creating an additional barrier for minority-spin electrons, which results in a reversal of the spin polarization.

Experimentally, the reversal of the SP associated with surface oxidation may be detected using spin-polarized STM measurements. ${ }^{11}$ Since the ferromagnetic tip is sensitive to the SP of the total local DOS above the surface (see, e.g., Ref. 29), the tunneling magnetoresistance in the system surface/vacuum/tip should change sign when the Co surface is oxidized. In other words, for the clean $\mathrm{Co}(111)$ surface the tunneling current should be higher when the magnetizations of the tip and the surface are aligned parallel (the dominating minority channel is then open), but for the oxidized surface it should be higher for the antiparallel configuration. 


\section{ACKNOWLEDGMENTS}

We are grateful to V. P. Antropov, W. H. Butler, P. H. Dederichs, J. C. Slonczewski, and N. E. Zein for useful discussions. This work was supported by NSF (Grant No.
DMR-0203359 and MRSEC Grant No. DMR-0213808) and the Nebraska Research Initiative. M.v.S. was supported by DARPA and ONR. Work at Sandia National Laboratories was supported by U.S. Department of Energy under Contract No. DE-AC0494AL85000.
${ }^{1}$ E.Y. Tsymbal, O.N. Mryasov, and P.R. LeClair, J. Phys.: Condens. Matter 15, R109 (2003).

${ }^{2}$ J.S. Moodera, L.R. Kinder, T.M. Wong, and R. Meservey, Phys. Rev. Lett. 74, 3273 (1995).

${ }^{3}$ J.M. MacLaren, X.-G. Zhang, W.H. Butler, and X. Wang, Phys. Rev. B 59, 5470 (1999).

${ }^{4} \mathrm{Ph}$. Mavropoulos, N. Papanikolaou, and P.H. Dederichs, Phys. Rev. Lett. 85, 1088 (2000).

${ }^{5}$ P. LeClair, H.J.M. Swagten, J.T. Kohlhepp, R.J.M. van de Veerdonk, and W.J.M. de Jonge, Phys. Rev. Lett. 84, 2933 (2000); P. LeClair, J.T. Kohlhepp, H.J.M. Swagten, and W.J.M. de Jonge, ibid. 86, 1066 (2001).

${ }^{6}$ C. Uiberacker and P.M. Levy, Phys. Rev. B 65, 169904 (2002).

${ }^{7}$ E.Y. Tsymbal and D.G. Pettifor, J. Phys.: Condens. Matter 9, L411 (1997).

${ }^{8}$ M. Sharma, S.X. Wang, and J.H. Nickel, Phys. Rev. Lett. 82, 616 (1999).

${ }^{9}$ J.M. De Teresa, A. Barthélémy, A. Fert, J.P. Contour, R. Lyonnet, F. Montaigne, P. Seneor, and A. Vaurès, Phys. Rev. Lett. 82, 4288 (1999); A. Barthélémy, A. Fert, J.P. Contour, F. Montaigne, and P. Seneor, Science 286, 507 (1999).

${ }^{10}$ I.I. Oleinik, E.Y. Tsymbal, and D.G. Pettifor, Phys. Rev. B 62, 3952 (2000).

${ }^{11}$ M. Bode, Rep. Prog. Phys. 66, 523 (2003).

${ }^{12}$ W. A. Harrison, Solid State Theory (McGraw-Hill, New York, 1970).

${ }^{13}$ I.I. Mazin, Europhys. Lett. 55, 404 (2001).

${ }^{14}$ S. Datta, Electronic Transport in Mesoscopic Systems (Cambridge University Press, Cambridge, UK, 1999).
${ }^{15}$ M. Jullière, Phys. Lett. A 54, 225 (1975).

${ }^{16}$ R. Meservey and P.M. Tedrow, Phys. Rep. 238, 173 (1994).

${ }^{17}$ J.C. Slonczewski, Phys. Rev. B 39, 6995 (1989).

${ }^{18}$ J.M. MacLaren, X.-G. Zhang, and W.H. Butler, Phys. Rev. B 56, 11827 (1997).

${ }^{19}$ J. Mathon and A. Umerski, Phys. Rev. B 60, 1117 (1999).

${ }^{20}$ E.Y. Tsymbal and D.G. Pettifor, Phys. Rev. B 58, 432 (1998).

${ }^{21} \mathrm{~S}$. Coleman, Aspects of Symmetry (Cambridge University Press, Cambridge, UK, 1985).

${ }^{22}$ M.I. Katsnelson, M. van Schilfgaarde, V.P. Antropov, and B.N. Harmon, Phys. Rev. A 54, 4802 (1996).

${ }^{23}$ I. Turek, V. Drchal, J. Kudrnovský, M. Sob, and P. Weinberger, Electronic Structure of Disordered Alloys, Surfaces and Interfaces (Kluwer, Boston, 1997).

${ }^{24}$ J. Kudrnovský, V. Drchal, C. Blaas, P. Weinberger, I. Turek, and P. Bruno, Phys. Rev. B 62, 15084 (2000).

${ }^{25}$ M. Methfessel, M. van Schilfgaarde, and R. A. Casali, in Electronic Structure and Physical Properties of Solids: The Uses of the LMTO Method, edited by H. Dreysse, Lecture Notes in Physics, Vol. 535 (Springer-Verlag, Berlin, 2000).

${ }^{26}$ O. Wunnicke, N. Papanikolaou, R. Zeller, P.H. Dederichs, V. Drchal, and J. Kudrnovský, Phys. Rev. B 65, 064425 (2002).

${ }^{27}$ M.C. Payne, M.P. Teter, D.C. Allan, T.A. Arias, and J.D. Joannopoulos, Rev. Mod. Phys. 64, 1045 (1992).

${ }^{28}$ E.Y. Tsymbal, I.I. Oleinik, and D.G. Pettifor, J. Appl. Phys. 87, 5230 (2000). Note that in this paper the SP was deduced from the local DOS in the vacuum without calculating the transmission function.

${ }^{29}$ S. Heinze, S. Blügel, R. Pascal, M. Bode, and R. Wiesendanger, Phys. Rev. B 58, 16432 (1998). 